\title{
Application of MPPC to granular electromagnetic calorimeter for ILC
}

\author{
Katsushige Kotera*† \\ Schinshu University, Faculty of Science, \\ Matsumoto, Nagano, Japan \\ E-mail: Coterraeazusa.shinshu-u.ac.jp
}

\begin{abstract}
MPPC has advantage to make a large and granular sensitive calorimeter. As an application of 1600 pixel MPPC, we constructed an electromagnetic calorimeter as a prototype for the International Linear Collider. The prototype calorimeter has an architecture of stacking 30 layers of scintillator pad paved with 72 finger size strip-schintillators. All 2160 strip-scintillators are individually readout by MPPCs. This compact calorimeter was tested with $1-32 \mathrm{GeV} / c$ electron and pion beam at Fermi National Accelerator Laboratory in 2008 and 2009. The structure and test results are presented.
\end{abstract}

International Workshop on New Photon Detectors PD09

June 24-26 2009

Shinshu University Matsumoto Japan

* Speaker.

${ }^{\dagger}$ representing the CALICE collaboration 


\section{Introduction}

The International Linear Collider (ILC) is expected to extend our knowledge of the elemental particle physics world beyond the current mostly reliable model called the standard model[1]. Requirement to realize this purpose is high precision measurement using the clear initial state and well reconstructed final state of the electron-positron collider. To determine the final state in quarks leptons and guage bosons, the reconstruction of jets is one on the key issues. A jet is a group of decay particles mostly originated from a quark being consist of many hadrons and electromagnetic particles in a small volume. One of the strategies to precisely reconstruct jets is combination of calorimetry and tracking, constructing individual particles within jets. In this method, called particle flow approach (PFLOW) [2], for example, the energy of a charged hadron is measured with tracking detectors, which has higher precision to determine the hadron energy than calorimeters. This method requires a highly granular calorimeter system to reconstruct individual particles, being combined with the information from the central tracking system. Figure 1 shows tracker and calorimeter with other parts of detector for ILC, which was proposed as one of ILC detector, named ILD[3]. The calorimeter is composed of two parts; electromagnetic calorimeter (ECAL) and hadronic calorimeter (HCAL). ECAL which measures the energy of electromagnetic particles with their showers is inside of HCAL. HCAL measures the energy of hadrons passing through ECAL.

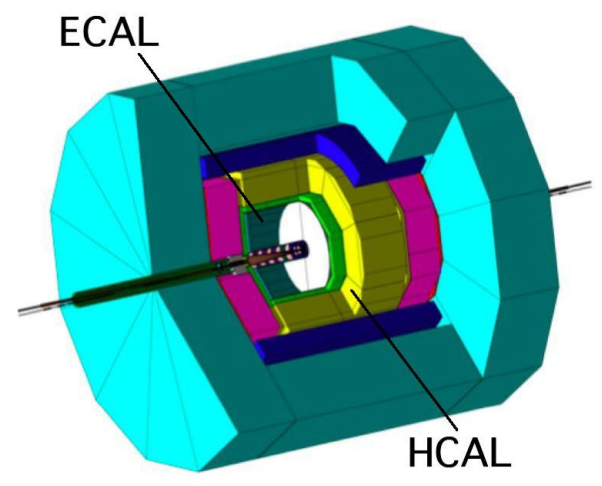

Figure 1: The ILD detector model. white volume is the tracker, green volume is ECAL, and yellow and purple are HCAL. The inner radius of ECAL is $1.85 \mathrm{~m}$ and the length of the barrel of ECAL is $4.9 \mathrm{~m}$.

According to the simulation studies to achieve the ILC physics, the granularity of ECAL should be $5 \mathrm{~mm} \times 5 \mathrm{~mm}-10 \mathrm{~mm} \times 10 \mathrm{~mm}$ segmentation of transverse dimension of jet direction and 25 - 30 layers of longitudinal segmentation for electromagnetic calorimeter [3]. For such granularity, there are two proposed technology of ECAL for ILD; Silicon tungsten ECAL (SiECAL) and scintillator tungsten ECAL (ScECAL). This manuscript is dedicated to introduce the ScECAL as an application of photon detection technology to make a granular calorimeter, focusing on a prototype module tested in September 2008 at Fermi National Accelerator Laboratory (FNAL).

SiECAL and ScECAL are being developed and studied by respective groups in the international CALICE Collaboration. 


\section{Requirements for ILD electromagnetic calorimeter}

Recently, ILD group released the Letter of Intent of ILD[3] based on intensive R\&D on all parts of detector. For the electromagnetic calorimeter, the inner radius of barrel should be 1850 $\mathrm{mm}$ and its longitudinal length is $4900 \mathrm{~mm}$. With two end cap parts, the total surface of ECAL should be $7.85 \times 10^{7} \mathrm{~mm}^{2}$. To achieve the state-of-the art PFLOW, the granularity of ECAL should be $5 \mathrm{~mm} \times 5 \mathrm{~mm}-10 \mathrm{~mm} \times 10 \mathrm{~mm}$ in transverse segmentation and $25-30$ layers longitudinal segmentation. Consequently, the number of channels of ECAL should be $2-9 \times 10^{6}$. For such unprecedented number of channels, each contribution should have complete robustness and low cost. The other requirement to the ILD calorimeter is to have the magnetic field tolerance, since the calorimeter is inside the $3.5 \mathrm{~T}$ magnetic field. In addition to satisfying these requirements, the performance of ECAL should be achieved showing the sufficient resolution, good linearity and uniformity of the energy response.

\section{Scintillator ECAL}

One of the candidates to answer these requirements discussed in the previous section is the ScECAL. This technique is realized by almost only the Pixelated Photon Detectors (PPDs) employed in order to readout the number of photons from scintillators. One more proposed special technique is using the strip shape scintillator shown in Fig. 2(1). The challenge is to make square granularity with alternately placed the longitudinal direction of strip scintillator explained in Fig. 2 (2).
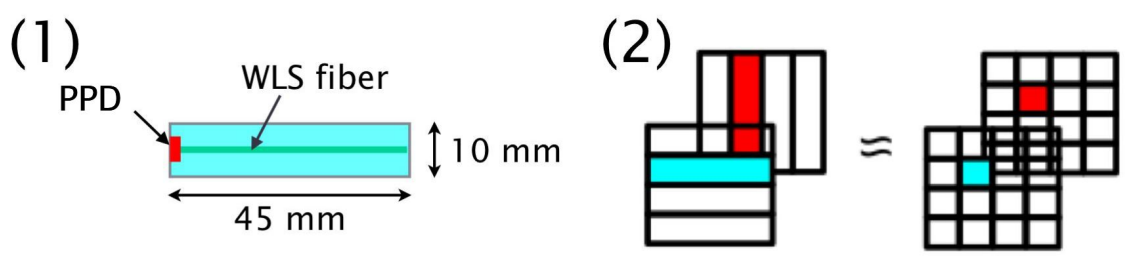

Figure 2: The strip scintillator with a PPD and a WLS fiber (1). Series of scintillators are alternately placed in the longitudinal directions (2).

\section{Prototype ScECAL}

\subsection{Design}

The ScECAL group has constructed a $180 \mathrm{~mm} \times 180 \mathrm{~mm}$ face size $\times 30$ layers prototype ECAL with 2160 scintillator strips, individually read by PPDs, and tested it in electron and hadron beams at FNAL. Figure 3 shows the structure of the prototype ScECAL and a photograph of one layer. Each layer is composed of scintillator layer and absorber layer of $3 \mathrm{~mm}$ and $3.5 \mathrm{~mm}$ thickness, respectively. 

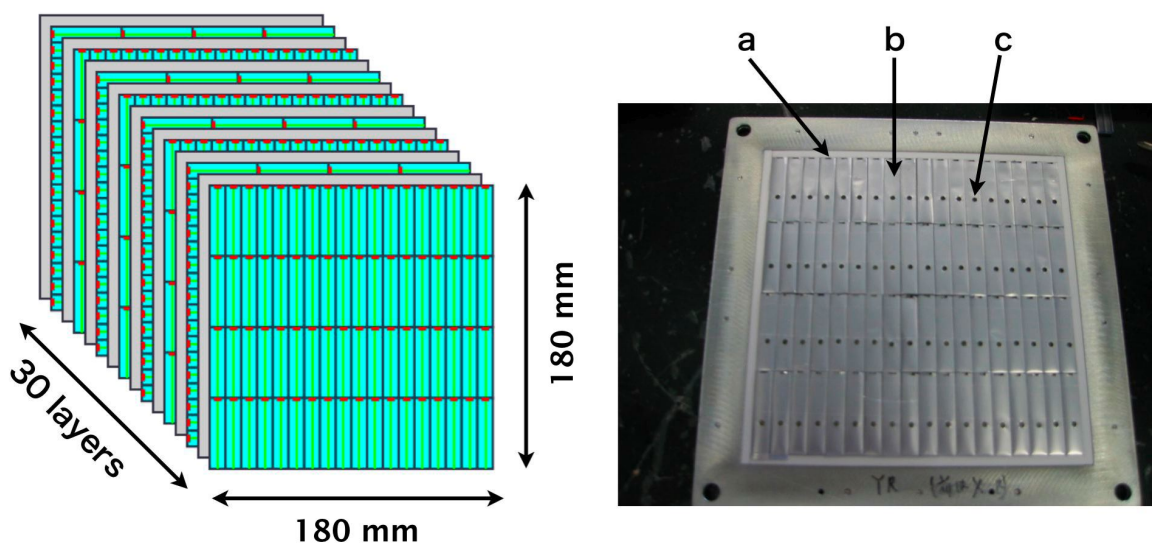

Figure 3: Right: structure of the ScECAL test module in Sep 2008 and April 2009 at FNAL. Left: picture of a sensitive layer showing the PPD housing (a), the scintillator strip hermetically covered by KIMOTO reflector (b), and holes to introduce LED light for the gain monitoring system (c) explained in Section 4.4.

A scintillator layer has $18 \times 4$ scintillator strips whose size is $45 \mathrm{~mm} \times 10 \mathrm{~mm}$ as shown in Fig. 3. In successive scintillator layers, the strips are alternately aligned vertically (" $\mathrm{X}$ " layers) and horizontally (" $\mathrm{Y}$ " layers). The scintillation photons are corrected by a wavelength shifting (WLS) fiber( Y-11M made by KURARAY Co., Ltd.) inserted along the longitudinal direction of center of each strip. The strip scintillators are made using extruded method by Kyungpook National University (KNU). Each strip scintillator is hermetically covered with the reflecting film made by KIMOTO Co., Ltd. The collected photons by WLS fiber are introduced into a PPD mounted in a housing in the end of each scintilltor strip. A PPD is soldered to a flat cable together with other eight PPDs. Signals from PPDs are fed into the readout baseboard through the flat cable.

The absorber layer is composed $88 \%$ tungsten, $12 \%$ cobalt and $0.5 \%$ lithium, and has Moliere radius of $10 \mathrm{~mm}$.

\subsection{Photon sensor PPD (MPPC) in prototype ScECAL}

One of the stable commercial products of PPD is MPPC by HAMAMATSU KK. The breakdown voltage, capacitance, and noise rate for each MPPC, series S10362-11-025MK were measured. This MPPC has 1600 pixel in $1 \mathrm{~mm} \times 1 \mathrm{~mm}$ square shown in Fig团(1). Figure 4(2) shows the distribution of capacitance of MPPCs and (3) shows the distribution of the breakdown voltage of MPPCs. The colors indicate the difference of obtained terms. All MPPCs in the ScECAL were supplied bias voltages of respective breakdown voltage $+3 \mathrm{~V}$.

\subsection{Sensor fiber connection}

In anticipation of the FNAL beam test, ScECAL group had a beam test at Deutsches ElektronenSynchrotron (DESY), with quarter size of current prototype ScECAL近 5, 6, 7. From this experiment, it is recognized that suppressing the non-uniformity of response of scintillator strip along to the longitudinal direction is crucial to keep small stochastic term of energy resolution. From another beam test at KEK to choose the best strip reflector condition and a bench test in a labo- 


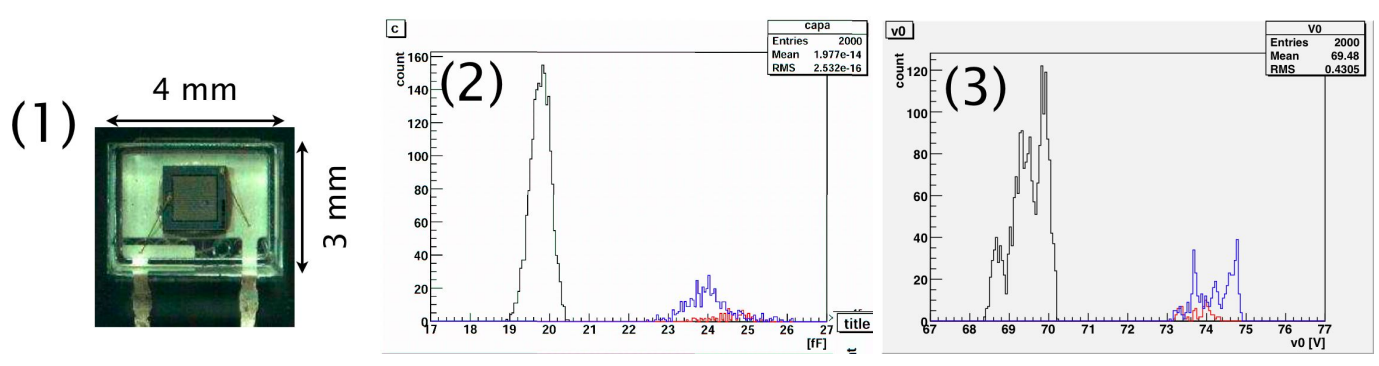

Figure 4: A photograph of MPPC (1), the distribution of capacitance of MPPC (2) and the distribution of breakdown voltage (3) of MPPC. In (2) and (3) black histograms are for MPPCs obtained in 2008 for FNAL beam test, and red and blue histograms are for MPPCs obtained in 2006. MPPCs in blue histograms are recycled from the beam test at DESY 2007.

ratory, it was recognized that the photons coming into MPPC not through WLS fiber increase the non-uniformity of response. Figure 5 explains the mechanism. The cross section of WLS fiber has one $\mathrm{mm}$ diameter round shape, while the sense area of MPPC has $1 \mathrm{~mm} \times 1 \mathrm{~mm}$ square shape. Therefore, some photons can come into MPPC not through WLS fiber but directly from the scintillator. The WLS fiber has long attenuation length enough. Thus, the response by the photons from WLS fiber does not have non-uniformity. However, since the attenuation length of extruded strip scintillators are not enough so far, the photons coming directly from scintillator increase the non-uniformity of response. To suppress this effect, a shade film was put between MPPC and scintillator. This shade has also another effect to reflect the photons going into the MPPC housing.

(1)

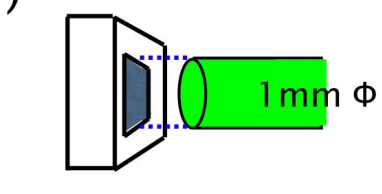

$1 \mathrm{~mm} \times 1 \mathrm{~mm}$ MPPC sensitive area

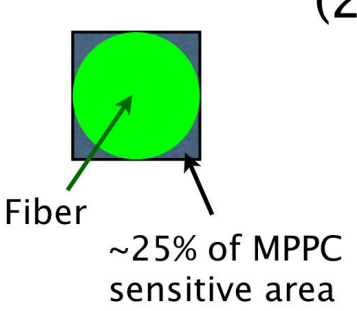

(2)

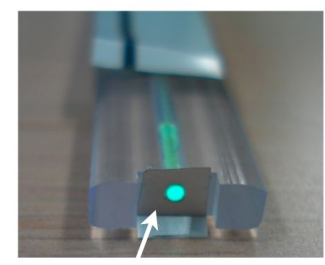

Shade

Figure 5: A schematic explaining the excess area of MPPC sensitive area (1), and the shade to reject photons coming into those excess area (2).

\subsection{LED calibration system}

The ScECAL have a gain monitoring system using LED light. An LED light is distributed through clear fibers, the fibers then distribute the light through notches on them. Figure 61) shows the fibers before they are installed on the ScECAL layers. As in Fig. 3 right, each strip has a hole on its reflector to take LED light from the notch. Figure 6(2) shows the fiber lays down along the row of the strip holes. 

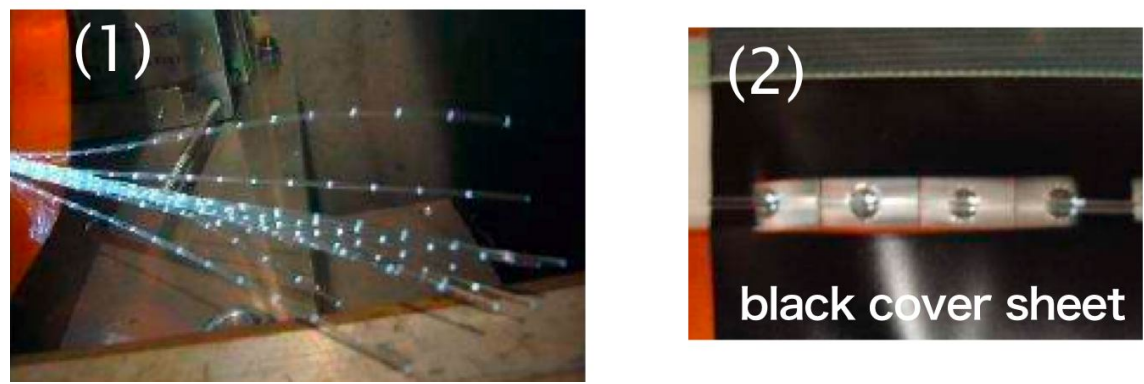

Figure 6: Clear fibers emitting LED light from notches on them (1), and a clear fiber laying along the holes on reflector of strips (2).

\section{Beam Test at Fermilab.}

\subsection{Goal of the Beam Test at FNAL September 2008 and May 2009}

Feeding back from the DESY beam test March 2007 and studies of bench tests, ScECAL had some modifications especially for the uniformity of response discussed in Section 4.3 and selection of the reflector and segmentation method of scintillators. In addition to evaluating these modifications, the goal of FNAL beam tests is the following;

- to establish comprehensive study of combined ScECAL and HCAL,

- to make the first evaluation of ScECAL performance,

- linearity of energy response for $1-32 \mathrm{GeV}$,

- enegy resolution for $1-32 \mathrm{GeV}$,

- effect of incident angle of particles,

- position dependence of response,

- to observe $\pi^{0}$ events and separation of two photons,

- to establish LED gain monitoring system.

In this manuscript, we dedicate to discuss the linearity and resolution of energy response with electrons and $\pi^{0}$ observations.

\subsection{Setup}

The beam test was performed at the MT6 experiment area in the Meson Beam Facility (MTBF) of FNAL. Electron and pion mixed beam with an energy between $1 \mathrm{GeV}$ to $32 \mathrm{GeV}$ is provided there. Putting iron dumper upstream of experimental region, the beam is converted into the muon beam. In the FNAL beam test, $32 \mathrm{GeV}$ muon beam runs were used for MIP calibration.

Setup of the beam line is shown in Fig. 7 Some combinations of trigger counters were used, which include the Ĉerenkov counter placed more upstream than the experimental site in Fig.7 For ScECAL, $200 \mathrm{~mm} \times 200 \mathrm{~mm}$ trigger counter pair was used for MIP calibration runs, and $100 \mathrm{~mm}$ 


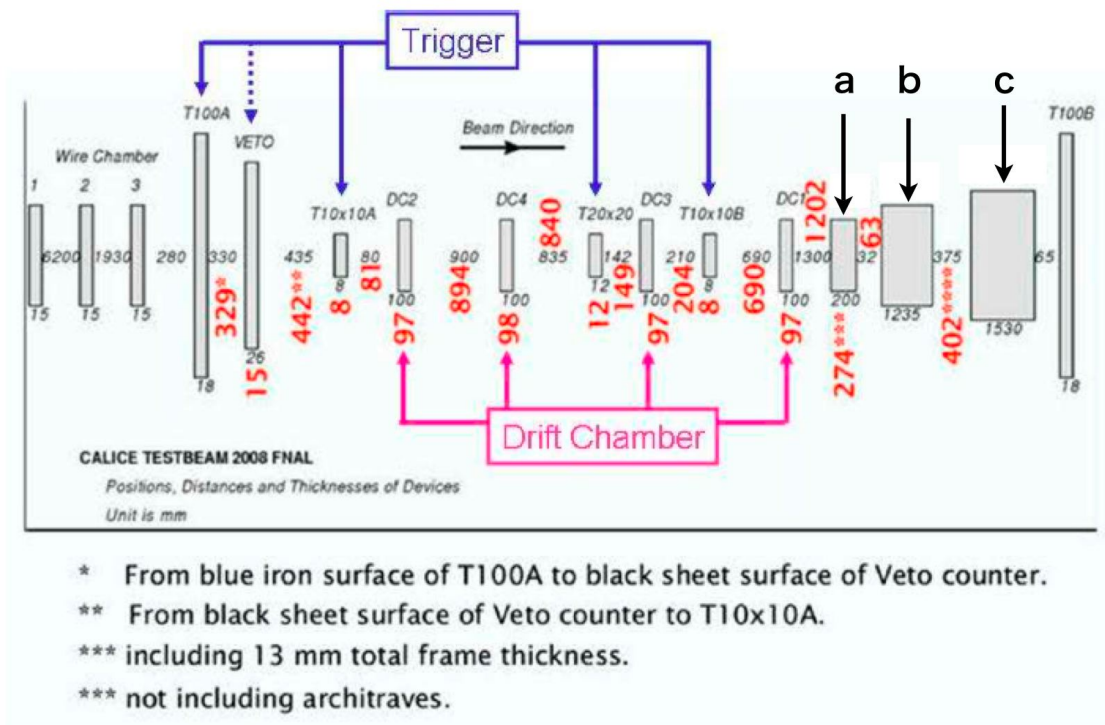

Figure 7: Configuration of the beam line at the MT6 in FNAL. a shows ScECAL, b shows HCAL and c shows the Tail catcher.

$\times 100 \mathrm{~mm}$ trigger counter pair was used for electron pion beam runs. The Ĉerenkov counter was used to select pions or electrons depending on the purpose of the runs.

\subsection{MIP calibration}

Each channel has different sensitivity from others due to the quality variation of each strip channel. Therefore, the calibration constant for each channel should be determined. Figure 8 (1) shows a typical distribution of the ADC counts for a channel in which a muon deposits MIP energy in each event. The MIP calibration constant for each channel is determined with the distance from the pedestal peak to the most probable value of the Landau function as the result of fitting to such distribution of ADC counts. The fitting Landau function is convoluted with a Gaussian function. The deposited energy in a channel is measured as the ADC counts subtracted by its pedestal value, then divided by the MIP calibration constant. Therefore, the deposited energies are counted in the number of MIPs. Figure 8(2) shows the distribution of the MIP calibration constant for 2160 channels. Since the gains of MPPCs are tuned to a common value more restrictively, such unexpected large width of the distribution will be discussed in Section 7

\subsection{PPD saturation correction}

Intrinsically, PPD has saturation of response, since it has a finite number of pixels and a pixel makes a certain response not depending on the number of incident photons. Figure 9 shows the MPPC saturation appearing as the deviation from linear as the function of PMT response, where the experimental setup is illustrated in Fig. 10. Such PPD saturation is corrected with a fitting results indicated with red curve in Fig. (9. The PPD response, $N_{\text {out }}$, is represented as a function of 
(1)

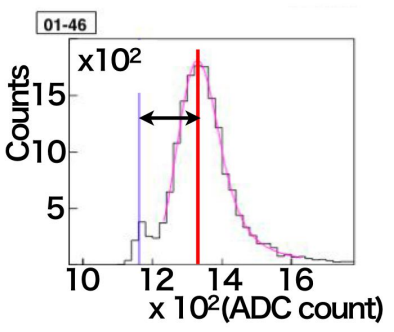

(2)

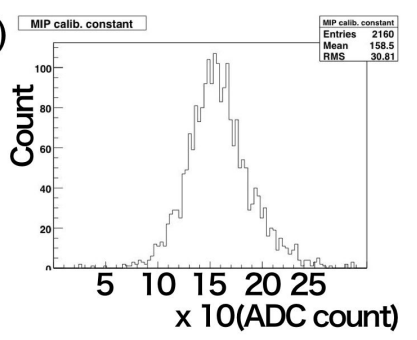

Figure 8: (1) A typical ADC charge distribution for MIP events in a channel. The purple line shows the fitting results by a Landau function convoluted with a Gaussian function. Blue line and red line indicate the place of pedestal peak and the most probable value of the Landau function, respectively. (2) The distribution of MIP calibration constants of 2160 channels.

real number of photons coming into the sensor, $N_{\mathrm{in}}$, as in the Eq. 5.1,

$$
N_{\text {out }}=N_{\text {pix }}\left\{1-\exp \left(\frac{-\varepsilon N_{\text {in }}}{N_{\text {pix }}}\right)\right\},
$$

where, $N_{\text {pix }}$ is the number of pixels in PPD and $\varepsilon$ is the photon detection efficiency. Because the photon generation in the scintillator strip has a time duration which allows some pixels to be active again, the number of pixels behaves to have more than actual number; 1600 for this experiment. Therefore, $N_{\text {pix }}$ is also a fitting parameter. With fitting result of $N_{\text {pix }}$ and $\varepsilon$, the revers function of equation 5.1 is used to make the correction of PPD saturation for each response.

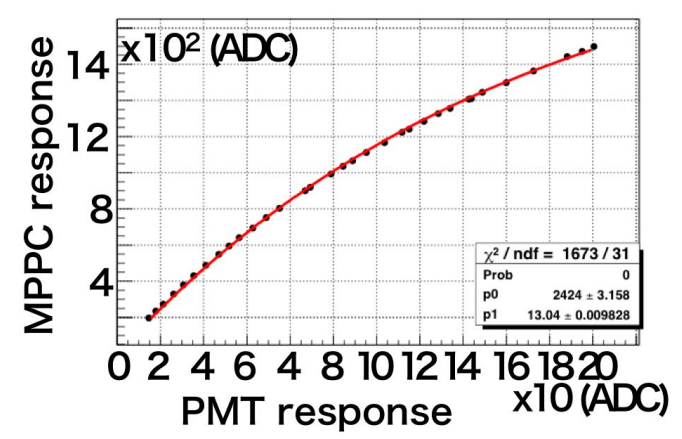

Figure 9: MPPC response mounted on a strip scintillator as a function of PMT response. The light source on the scintillator is $400 \mathrm{~nm}$ picosecond laser pulse.

\section{Results}

\subsection{Linearity and resolution of energy response}

Figure 11 shows the energy spectra of deposit energy in the ScECAL for 1, 3, 6, 12, 16, 25, $32 \mathrm{GeV}$ electron beams. From these spectra, the linearity and the resolution of energy response are induced. 


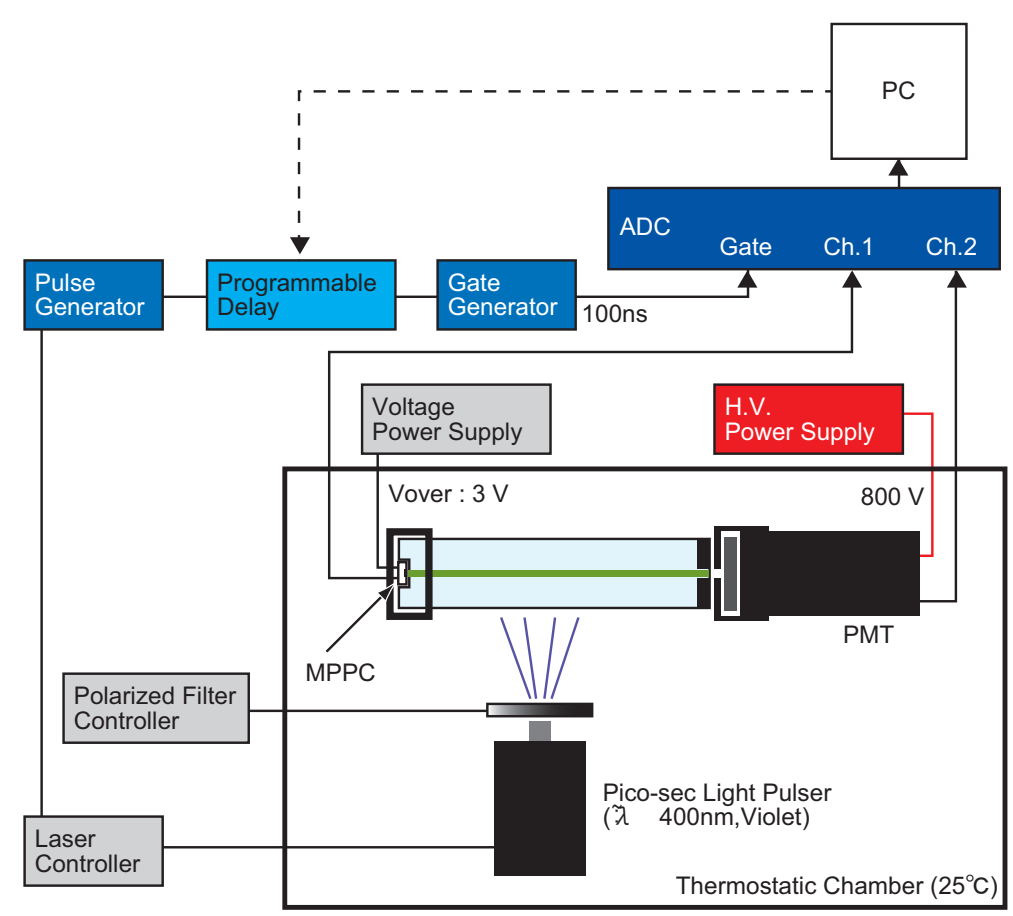

Figure 10: Setup of the response curve measurement using pico-second laser system, the scintillator strip and the MPPC.

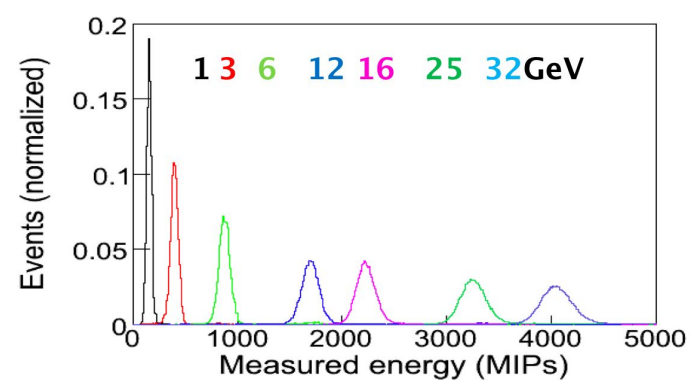

Figure 11: The energy spectra for 1, 3, 6, 12, 16, 25, $32 \mathrm{GeV}$ electron beam in September 2008 at FNAL, MT6.

Figure 12 (1) shows the deposit energy in the ScECAL represented in the number of MIPs as the function of the momentum of incident electrons. The linear fitting shows the good linearity. To make numerical evaluations for the linearity, the ScECAL group is working in the progress.

Figure 12 (2) shows the energy resolutions of deposit energy in the ScECAL as the function of the inverse of the square root of the momentum of incident electrons. An energy resolution is measured as the sigma divided by the mean of energy spectrum. Although we are still working to make openable values, the plots show that the energy resolution of ScECAL is reasonable.

In both plots each dot is distinguished in three colors; red: the results from the events in beam pointing on the center of the front face of ScECAL, blue: the results from the events in beam pointing on the $22.5 \mathrm{~mm}$ from the center in both $\mathrm{X}$ and $\mathrm{Y}$ direction, and black: the results from 
both events. Since the near PPD sides and the far PPD sides are closely exist in the center region, it has variable response in that region, when each strip has non-uniformity depending on the distance from the PPD. On the other hand, on $22.5 \mathrm{~mm}$ from center, it is middle place for every strips. Therefore, even if there are non-uniformity of response, the response on this region, called uniform region, is stable. According to the result of DESY beam test 2007, when there is non-uniformity in each strip, the difference between two region appears especially in the stochastic term of energy resolution. There is no clear difference of energy resolution especially on large momenta. This indicates that the uniformity of the strips is improved in current ScECAL.

(1)

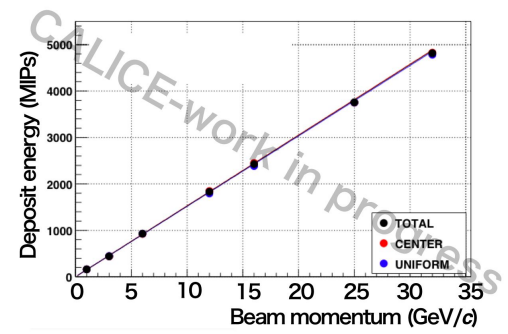

(2)

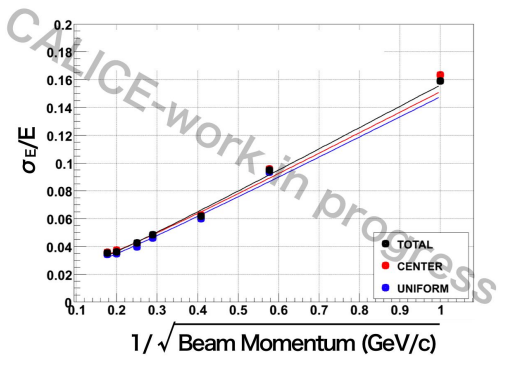

Figure 12: The measured deposited energy in ScECAL as a function of beam momentum. Solid line shows the result of linear fitting (1), and The energy resolution of ScECAL as a function of reverse of square root of the beam momentum (2).

\section{2 $\pi^{0}$ events}

PFLOW requires separation of clusters in the calorimeter. $\pi^{0}$ events provide us a good practice to separate two close photons. To create $\pi^{0}$ events, $10^{6} \pi^{-}$beams were pointed into the iron target located in the beam line $1.85 \mathrm{~m}$ upstream of the front face of ScECAL. The iron target has $60 \mathrm{~mm}$ thickness, and the energy of $\pi^{-}$were $16-32 \mathrm{GeV}$. The distance of $1.85 \mathrm{~m}$ corresponds to the inner radius of ECAL barrel of ILD. Figure 13 shows the distribution of invariant mass reconstructed from two cluster events, where the photon energies are obtained based on the energy-MIP relation shown in Fig.12(1). A peak appeared near $130 \mathrm{GeV} / \mathrm{c}^{2}$, however it is rather smaller than the world average of $\pi^{0}$ mass.

\section{Discussions and future directions}

The ScECAL showed the totally expected results. However, more detail with quantitative evaluations are expected. The ScECAL group is working on progress to get the deviations of energy deposits from the linear fitting line, the energy resolution represented in the stochastic term and constant term of the fitting results to the plot in Fig. 12 (2), and the value of reconstructed $\pi^{0}$ mass.

The distribution of calibration constants was unexpectedly broad. The variations from the fluctuation of bias voltage are ignorable, because the bias voltages were individually controlled for every PPDs in $\pm 0.1 \mathrm{~V}$ to have same gain. One of the ideas is the quality of PPD-WLS fiber 

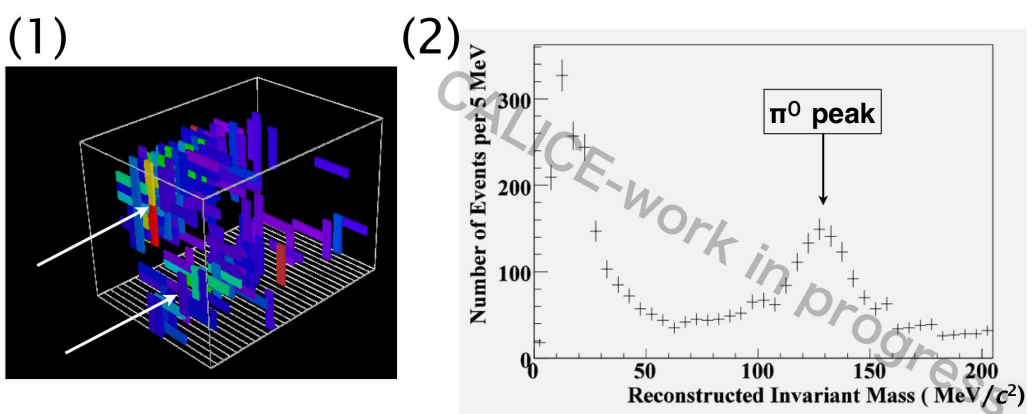

Figure 13: An example of two gamma event by $\pi^{0}(1)$, and the reconstruction mass of such events (2).

matching. Although we succeeded to modify the uniformity of response in each strip using the shade in front of PPD, we leave the control of the spacial matching between PPD and WLS fiber. Difference PPD-WLS fiber matching can make the difference calibration constant. The effect of the variations of calibration constants on the energy resolutions should be investigated.

The data takings were successfully achieved in September 2008 and May 2009 for the goals we discussed in 5.1, ECAL/HCAL combined performance, effect of incidental angle of particles, and position dependence of response. The group is working on progress for those goals.

The scintillator width in current ScECAL is $10 \mathrm{~mm}$. The ScECAL group is further studying to optimize the width and length to get best performance, simultaneously developing the clustering algorithm to evaluate the strip type scintillator calorimeter with PFLOW.

\section{Summary}

The ScECAL group in the CALICE collaboration proposes the strip scintillator calorimeter using PPDs to achieve the granular electromagnetic calorimeter for ILD. A prototype ScECAL was tested with $1-32 \mathrm{GeV} / c$ electron and pion beam at FNAL September 2008 and May 2009. The data shows expected performance on the linearity and resolution of energy response. The ScECAL group is continuously working on the analysis to get openable and quantitative evaluations.

\section{References}

[1] J. Brau et al., International Linear Collider reference design report, Vol 1: Executive summary, ILC-REPORT-2007-001 (2007).

[2] M. A. Thomson, "Progress with Particle Flow Calorimetry" arXiv:0709.1360[physics.ins-det].

[3] ILD Concept Group, "The International Large Detector Letter of Intent" (2009).

[4] CALICE Collaboration, CALICE Analysis Note 005 (2007).

[5] CALICE Collaboration, CALICE Analysis Note 006 (2007).

[6] CALICE Collaboration, CALICE Analysis Note 007 (2008).

[7] CALICE Collaboration, CALICE Analysis Note 012 (2008). 